Meeting Report

\title{
Cell death mechanisms: life in the balance
}

\author{
A Hague $^{\star, 1}$ and A Verkhratsky ${ }^{2}$ \\ Cell Death and Differentiation (2009) 16, 512-514; doi:10.1038/cdd.2008.157
}

Cell Death Mechanisms Meeting by the European Tissue Culture Society (UK branch) held at University of Bristol on 23 April 2008.

This report summarizes a 1-day meeting on Cell Death Mechanisms organized by the European Tissue Culture Society (UK branch) held at University of Bristol on 23 April 2008. The meeting brought together scientists interested in mechanisms of neuronal and glial survival and degeneration with those interested in reactivating or mimicking cell death signals to treat cancer.

David Rubinsztein (Cambridge) described how upregulating autophagy protects against neurodegeneration in diseases caused by aggregate-prone mutant proteins. Macroautophagy is a bulk degradation process involved in the clearance of long-lived proteins, protein complexes and organelles, in which autophagosomes containing these constituents fuse with lysosomes where the contents are degraded by acidic hydrolases. Huntington's disease is caused by a polyglutamine-expansion mutation in the huntingtin protein that makes it toxic and aggregate prone. Of the two main pathways to protein clearance in mammalian cells - the ubiquitin-proteasome and the autophagy-lysosome pathways, the latter is thought to be the better therapeutic target, as the proteasome has a narrow opening and cannot accommodate polymers. As proof of principle, the mTOR inhibitor rapamycin activates autophagy and enhances the clearance of polyglutamine proteins in cell culture and in Drosophila eye models, protecting against cytotoxicity. A rapamycin ester is favoured over rapamycin for clinical use, and in mouse models this resulted in much smaller and fewer aggregates in the brain than a placebo control and a marked improvement in a range of behavioural correlates of disease. Autophagy protects against other aggregate proteins such as ataxin 3 (mutated in spinocerebellar ataxia type 3) and tau mutants that cause temporal dementia/tauopathy. Therefore it appears that autophagy is important for clearing protein aggregate-prone proteins, and upregulating autophagy is helpful in these diseases.

Recently, from drug screening experiments to identify mTOR-independent autophagy-inducing drugs, L-type $\mathrm{Ca}^{2+}$-channel antagonists such as verapamil, the $\mathrm{K}^{+}$ATPchannel opener minoxidil, and the $G(i)$ signalling activator clonidine have been shown to induce autophagy. These drugs act to decrease intracytosolic calcium or cAMP levels and impact on a pathway, where myo-inositol-1,4,5-triphosphate $\left(\mathrm{InsP}_{3}\right)$ releases $\mathrm{Ca}^{2+}$ from the endoplasmic reticulum, activating calpains, which in turn, cleave and activate $\mathrm{G}(\mathrm{s}) \alpha$ (thus increasing cAMP levels) and inhibit autophagy. A further potential feed forward loop occurs in which cAMP regulates Ins $\mathrm{P}_{3}$ levels, releasing endoplasmic reticulum (ER) $\mathrm{Ca}^{2+}$ and enhancing calpain activity, activating $\mathrm{G}(\mathrm{s}) \alpha$, which in turn regulates cAMP levels. So, insults that elevate cytosolic $\mathrm{Ca}^{2+}$ inhibit autophagy, thus retarding clearance of aggregateprone proteins. This may lead to an array of new candidate drugs for many incurable neurodegenerative diseases.

Alexei Verkhratsky (Manchester) reminded us that the glial cells form $90 \%$ of the human brain, whereas interestingly they are much less numerous in other species. Glial cells are central in maintaining brain homeostasis and are represented by astrocytes, oligodendrocytes and microglial cells. Each of these subtypes expresses a variety of neurotransmitter receptors, many of which, when activated by neural activity, initiate glial $\mathrm{Ca}^{2+}$ responses, which produce interglial $\mathrm{Ca}^{2+}$ waves, that in turn activate release of 'glio' transmitters (which include glutamate, ATP, taurine, D-serine and probably many others) that can signal back to neurones, thus functionally integrating neuronal and glial circuitries. Astrocytes can form an extended physically connected syncytium. In brain pathologies, glial scars serve to fence off damaged areas. Within these areas, glutamate release from damaged cells is cytotoxic, and in stroke, death signals from the infarction lead to a propagated wave of cell death. It is believed that the glial cells deal with the damage by sealing and eliminating the damaged area to protect the rest of the brain. Atrophy of the glia is a feature of Alzheimer's disease and other dementias and can be important in driving disease progression.

Pierluigi Nicotera (Leicester) took up the story. Although $\mathrm{Ca}^{2+}$ signals are necessary for cell communication and survival, abnormal cellular $\mathrm{Ca}^{2+}$ load can trigger different cell death programs depending on variety of factors, such as energy requirement, signalling molecules, differentiation status or the intensity of the insult. During excitotoxicity, local glutamate-driven $\mathrm{Ca}^{2+}$ overloads at the postsynaptic areas

${ }^{1}$ Department of Oral and Dental Science, University of Bristol, Bristol, UK and ${ }^{2}$ Faculty of Life Sciences, University of Manchester, Manchester, UK ${ }^{*}$ Corresponding author: A Hague, Department of Oral and Dental Science, University of Bristol, Lower Maudlin Street, Bristol BS1 2LY, UK.

Tel: + 44117 3424114; Fax: 44117 3424428; E-mail: a.hague@ bristol.ac.uk 
predominantly elicit apoptosis, whereas global $\mathrm{Ca}^{2+}$ signals induced by widespread excess glutamate cause necrosis. $\mathrm{Ca}^{2+}$ enters via NMDA glutamate receptors and also through other $\mathrm{Ca}^{2+}$-permeable channels such as TRPM7 and ASICs. Long-lasting increases in $\left[\mathrm{Ca}^{2+}\right]_{\mathrm{i}}$ stimulate calpains, which cleave sodium calcium exchanger $\mathrm{NCX} 3$, thus reducing $\mathrm{Ca}^{2+}$ clearance. Unregulated calcium-dependent proteolysis is a key process during excitotoxicity and suppression of calpains ameliorates ischaemic damage. Before the cells die, the mitochondria take up huge amounts of calcium acting as a cytoprotective buffer. ER $\mathrm{Ca}^{2+}$-induced $\mathrm{Ca}^{2+}$ release (CICR) mediated by the ryanodine receptors (RyR) is essential for synaptic plasticity. Activation of $\mathrm{Ca}^{2+}$-permeable nicotinic acetylcholine receptors induces $\mathrm{CICR}$; released $\mathrm{Ca}^{2+}$ increases CREB phosphorylation, which promotes upregulation of RyR type 2. PhosphoCREB binds to a regulatory element for the RyR2 gene to amplify the CICR and synaptic activity that prolongs CREB phosphorylation.

The next session moved to considering the p53 family of proteins. Xin Lu (Oxford) updated us on the ASPP family of p53 regulators. This family consists of ASPP1, ASPP2 and iASPPP. ASPP1 and 2 stimulate the apoptotic function of p53 whereas iASPP inhibits the apoptotic function of p53. This function is mediated by alterations in p53-promoter selectivity depending on which ASPP protein is bound. For example, ASPP 1 and 2 direct p53 to activate genes-encoding apoptotic proteins, Bax and PIG3, but in this complex, p53 has little effect on $\mathrm{p} 21^{\mathrm{WAF}-1}$ transactivation. The mouse knockout for Aspp2 illustrates that this is a haploinsufficient tumour suppressor, as the mice get tumours faster than wild-type mice. ASPP2 cooperates with $\mathrm{p} 53$ to suppress tumour growth in vivo, explaining why all ASPP2 contact residues of p53 were mutated in human cancer with a relatively high frequency. Breast carcinomas show low levels of p53, ASPP1 and ASPP2 and some have elevated levels of iASPP. ASPP1 and 2 are frequently methylated and this correlates with poor prognosis.

Gerry Melino (Leicester) talked on p63, the progenitor of the p53 family that 'brings us from cell death back to stem cells'. p63 has only one-third the capacity of p53 to induce apoptosis. p63 is transcribed from two different promoters, generating two classes of proteins: one containing an N-terminal transactivation domain (TAp63) and another that lacks this domain $(\Delta \mathrm{N})$. The TAp63 knockout mouse has a very strong phenotype and is born without a differentiated epidermis, with craniofacial and limb malformations. Similarly, p63 mutations are the cause of a group of human syndromes characterized by cleft lip and palate, loss of teeth, ectodermal dysplasia and loss of the epidermis, as well as mammary gland hyperplasia.

p63 is critical for differentiation and programmed cell death in epidermal keratinocytes, where the cell death is a tissuespecific form of death distinct from apoptosis, but is the contribution of p63 to this process because of induction of differentiation, failure of cell death or suppression of stem cell behaviour? Gerry highlighted the work of Elaine Fuch's group illustrating that the $\triangle N p 63 \alpha$ mRNA is targeted for degradation by the microRNA miR-203. Hence, miR-203 and p63 expression inversely correlate in the skin. miR-203 functions to inhibit proliferation of keratinocytes and is induced in the skin concomitantly with stratification and differentiation because of this. Loss of miR-203 may prove to be important in cancer, as it may prevent cell-cycle exit during differentiation. Expression of proliferative markers such as p63, SOCS3 and Zfp281 is inhibited by miR-203. In response to UVC irradiation, miR-203 levels increase and p63 levels decrease; hence, miR-203 may also be involved in cell-cycle arrest in response to UVC irradiation, and may affect the cell's decision between arrest and apoptosis. Gerry's group is developing small molecule inhibitors of Itch to prevent p63 degradation with a view to upregulating apoptosis and chemosensitivity.

Moving to therapeutic utilization of our knowledge of cell death signalling, Gerry Cohen (Leicester) explained how primary chronic lymphocytic leukaemia (CLL) cells respond to the BH3-only protein mimetic ABT-737 developed by Abbot Inc. This molecule is designed to sit in the hydrophobic groove of Bcl-xL, Bcl-2 and Bcl-w. It does not inhibit Mcl-1. ABT-737 induced remarkably efficient and rapid death of these cells. All the usual features of apoptosis were there - Bax translocation to the mitochondria, caspase cleavage, PARP cleavage, phosphatidylserine exposure, sub- $\mathrm{G}_{1}$ peaks on flow cytometric analysis of DNA content and chromatin condensation. However, in addition to these classical hallmarks of apoptosis, swelling of the mitochondrial matrix, and discontinuities in the outer mitochondrial membrane were observed. Normally these would be considered to be features of necrosis rather than apoptosis, and this seemed to be independent of caspases. Mitochondrial inner membrane permeabilization was confirmed using calcein AM/cobalt staining. This was shown to be independent of permeability transition pore opening by use of PTP inhibitors, which failed to block release of the mitochondrial stain. Other primary cells (marginal zone lymphoma; mantle cell lymphoma and follicular lymphoma) also responded similarly to ABT-737) but very interestingly, cell lines did not. These died by classical apoptosis without the breaks in the outer mitochondrial membrane. There are fundamental differences between primary CLL cells and the cell lines, as Gerry illustrated. For example, Bcl-2 expression is much higher in primary CLL cells than it is in cell lines. As obtaining good knockdown of Bcl-2 in CLL cells is difficult, we may have to wait to find out whether this is the reason for the difference in response, but this clearly means that our work with cell lines should always be viewed with caution. For clinical application, this work gives real optimism. A new orally active analogue, ABT-263, is now being developed for use in the clinic.

Keeping with the mitochondrial involvement in apoptosis, Seamus Martin (Dublin) discussed whether mitochondrial fission is important in cytochrome $c$ release. Mitochondria are long and tubular in HeLa cells, but during apoptosis they fragment and the mitochondrial network collapses. This is caspase independent and occurs relatively early stage in apoptosis. The drp1 protein translocates to mitochondria and makes a spring-like coil around them, causing them to constrict and fragment. Mitofusins 1 and 2 (large GTPases) regulate mitochondrial fusion. Overexpression of a drp1 mutant (K38A) or the mitofusions did not delay apoptosis and cytochrome $c$ release was not affected. By contrast, overexpression of $\mathrm{Bcl}-\mathrm{xL}$ as a positive control prevented apoptosis but did not block Bax-induced mitochondrial fission. 
Interestingly, $\mathrm{Bcl}-\mathrm{xL}$ promotes mitochondrial fission and fusion, which Seamus suggests is because $\mathrm{Bcl}-\mathrm{xL}$ neutralizes both Bax and Bak, whereas Mcl-1 does not because it is only able to neutralize Bak, and it can only promote fusion and fission if Bax and Bak levels are reduced. In Caenorhabditis elegans, the homologue of Bcl-2, CED-9, is localized to mitochondria - but why? There is no cytochrome $c$ involvement in apoptosis signalling in the nematode. We know that Bcl-2 substitutes for CED-9 in the nematode, so the question was what would CED-9 regulate in mammalian cells? In fact, CED-9 promoted dramatic remodelling of the mitochondrial network in a variety of mammalian cell types by inducing mitochondrial fusion. However, CED-9 failed to antagonize Bax-induced cytochrome $c$ release or apoptosis. So, in addition to regulating CED-4/CED-3-dependent programmed cell death, it appears that CED-9 may also participate in the regulation of mitochondrial fusion/fission dynamics, a function that has been conserved in mammalian Bcl-2 family proteins. Hence, Bax/Bak-induced fragmentation of mitochondrial networks and cytochrome $c$ release are separable events.

Simon Cook (Babraham, Cambridge) illustrated how the $\mathrm{BH}$-only protein Bim is regulated by cell-survival signalling through the Raf-MEK-ERK1/2 pathway. Bim is important in cell death following serum withdrawal. Serum starvationinduced cell death is inhibited by cycloheximide, suggesting that this requires new protein synthesis; indeed, there is an increase in Bim protein levels early, following serum withdrawal. Bim ${ }^{-1-}$ MEFs exhibit a $90 \%$ decrease in caspase activation compared to wild-type cells, but ultimately another BH3-only protein or another cell death pathway kicks in and lack of Bim provides only a transient protection against cell death. Of the three splice variants of Bim, the most common, Bim $_{E L}$, is phosphorylated by ERK $1 / 2$, which promotes its polyubiquitination and degradation by the proteasome. A useful tool to study the importance of this in cell survival is the inducible Raf-1/ER construct, which can be switched on by 4-hydroxytamoxifen to give strong induction of the MEK-ERK

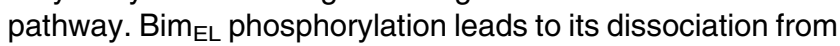
MCL-1, making it more susceptible to turnover. The E3 ligase that targets Bim $_{E L}$ is as yet unidentified, but in contrast to previous reports does not appear to be $\mathrm{Cbl}$. The mechanism of Bim $_{\mathrm{EL}}$ degradation is important to determine, as many tumour cells have very low levels of Bim, and its stabilization may prove to be a useful therapeutic strategy.

B-Raf mutations lead to constitutive ERK1/2-dependent Bim $_{E L}$ turnover and tumour cells containing these mutations appear to become 'addicted' to ERK1/2 signalling for survival. AZD6244, a novel MEK1/2 inhibitor that is in clinical trials, overcomes growth factor-independent cell survival in colorectal cancer cells with a B-Raf ${ }^{\mathrm{V} 600 \mathrm{E}}$ mutation consistent with reports that it can promote regression of such tumours when grown as xenografts. What about K-Ras mutant colorectal carcinomas? K-Ras does not signal just through ERK - in some colorectal cancer cell lines K-Ras signals strongly to ERK and in others much less, so the simple presence of a $\mathrm{K}$-Ras mutation is not predictive of ERK-dependent regulation of Bim or sensitivity to MEK inhibitors. However, although $\mathrm{K}$-Ras can activate other effector pathways involved in Bim regulation, such as the phosphoinostide 3-kinase (PI3K)-PKB pathway, even colorectal cancer cells with K-Ras mutations and/or mutations in the PIK3CA gene encoding the p110 subunit of PI3K are dependent on ERK1/2 for repression of Bim and cell death if they exhibit high levels of ERK1/2 signalling. MEK inhibitors have relatively less impact on cells that exhibit activation of both Raf and PI3K pathways; in such cases inhibitors against both pathways may be the most effective option.

Julian Downward (CR-UK London Research Institute) spoke further on the integration of type I PI3K- and Rassignalling networks in regulating cell survival and their role in oncogenic transformation. Ras is of significant interest because of activating mutations in the GTP-binding site of around $20 \%$ of human tumours. Ras does not immortalize cells, but contributes to the other five characteristics of cancer cells as defined by Hanahan and Weinberg. Ras interacts with the p110 subunit of PI3K in development and in tumorigenesis. The PIK3CA gene has activating mutations in $10-15 \%$ of human cancers. Another mechanism of activation is the allosteric catalytic activation of PI3K by Ras. Julian's laboratory used point mutation at Tyr208 and Tyr232 of the $\mathrm{p} 110 \alpha$ subunit in mice to block its ability to interact with activated Ras. Interestingly, loss of Ras interaction with $\mathrm{p} 110 \alpha$ in MEFs compromises the activation of PI3K by EGF, but not by PDGF. Mice homozygous for the $\mathrm{p} 110 \alpha$ mutation exhibit very dramatically reduced rate of cancer incidence in two models of Ras oncogene-driven tumour formation, Tyler Jacks's K-ras mouse that is prone to lung cancer and Alan Balmain's mouse skin model. Failure of Ras to engage PI3K results in elevated rates of apoptosis in tumour precursor lesions and consequent failure of tumours to develop. Targeting this interaction by pharmacological intervention could be a way of inducing apoptosis in tumours.

Another approach taking Julian's laboratory is to use large-scale RNA interference libraries corresponding to 21000 genes to identify targets that contribute to the Ras pathway addiction, comparing the apoptotic response to knockdown in K-Ras mutant and wild-type colorectal cancer cell line pairs. A number of pathways have been identified in addition to the MAPK-signalling pathway and these include purine metabolism, RNA polymerases and proteosomal degradation pathways. Drugs that selectively target Ras transformed cells include proteasome inhibitors and inhibitors of transcription, in particular the topoisomerase inhibitors such as etoposide and camptothecin. Although the mechanism is still to be determined, the way in which cells respond to DNA damage is fundamentally different in Ras-transformed cells. Although these agents induce apoptosis, they are still only half as effective as Ras siRNA, and the challenge is to optimize therapeutic targeting of Ras-driven survival pathways.

Despite different primary interests of the speakers, we discovered many links between results and ideas coming out of different laboratories. 